Research Round Up- Managing Diabetes Care in the COVID-19 Pandemic

Introduction

The last research round up provided you with an overview of recent research around remote healthcare in COVID-19 with a focus on remote consultation and prescribing. This month we will continue the theme of prescribing and care management during the COVID-19 global pandemic with a focus of prescribing for diabetes. As we are in the grip of a second wave of the pandemic and have seen increasing pressure on the NHS it is important to keep abreast of management of chronic longterm conditions such as diabetes so that disease progression and complications can be monitored. Many of you are involved in managing long term conditions and although the research presented here is centred on diabetes, many of the issues and points are transferable to other chronic longterm conditions.

Effect of remote management on comprehensive management of diabetes mellitus during the COVID-19 epidemic

This piece of original research, carried out in May 2020 in China used a questionnaire method to gather information about diabetic patients as a measure to determine recruitment before conducting a randomized controlled study to confirm the effectiveness of remote management using the mobile phone WeChat app on comprehensive management of diabetes mellitus during the COVID-19 epidemic. 540 valid questionnaires were completed. The results of the questionnaire survey showed that the management of diabetes during the epidemic was difficult and lead to a single centre trial being recruited to.

The researchers assigned 90 cases to the intervention group (use of WeChat) and 90 cases to the control group. The intervention group was managed remotely through the WeChat app, and the control group received traditional medical treatment. The blood glucose, blood pressure, body mass index (BMI), time in range (TIR) and incidence of hypoglycaemia were compared after three months of follow-up. Due to some drop outs and exclusions, 80 people from each group had their data analysed. The demographics of the groups were as follows, the percentage of males was $63.3 \%$ and that of females was $36.7 \%$. The majority of patients were $40-60$ years old, accounting for $53.3 \%$. Patients with T2DM were the most, accounting for $90.6 \%$. The duration of diabetes was 1-5 years in $45.6 \%$ of patients.

The results outlined by the researchers showed that BMI and postprandial blood glucose (PBG) of the control group at 3 months was significantly higher than that at baseline $(P<0.001)$, and TIR decreased at 3 months $(P<0.05)$. There was no significant difference in blood pressure compared with baseline in the control group, while blood pressure decreased in the intervention group $(P<$ $0.05)$. In the intervention group, fasting blood glucose (FBG) and PBG decreased compared with their baseline values, and the TIR level increased, both of which were statistically significant $(P<0.001)$. The FBG, PBG, and TIR of the intervention group were better than those in the control group at 3 months $(P<0.05)$. There was no difference in the incidence of hypoglycaemia between the two groups. The researchers conclude from these results that remote management can increase TIR without increasing the risk of hypoglycaemia. Remote management can prevent weight gain and improve patients' self-management and compliance during the COVID-19 epidemic. They recommend that through the supervision and encouragement of doctors, patients can avoid weight gain and improve self-management ability and compliance during the COVID-19 epidemic. 
https://www.sciencedirect.com/science/article/pii/S1751991820303636

Inpatient diabetes care during the COVID-19 pandemic: A Diabetes UK rapid review of healthcare professionals' experiences using semi-structured interviews

This article published in October 2020 in the Journal of Diabetic Medicines, is a rapid review conducted by Diabetes UK consisted of a series of semi structured interviews of 28 healthcare staff. These healthcare professionals interviewed were made up of diabetes consultants, diabetes specialist nurses and any allied health professionals that were involved in delivery of diabetes inpatient care. The aim of the study was to review inpatient care for people with diabetes with a view to improvement. The COVID-19 pandemic has impacted the way care is delivered across the UK. Diabetes UK sought to understand how inpatient care for people with diabetes has been affected and to identify opportunities, areas of concerns and recommendations for future practice.

The authors found that although there was disruption to inpatient diabetes services this, for the most part, created positive environments and opportunities for new ways of working (in 93\% of trusts included), but in the minority (the remaining 7\%), impacted on the quality of care clinicians felt they were able to deliver. Regardless of location, the way hospitals re-organised and prioritised inpatient services frequently determined whether clinicians reported a positive or negative experience. Services already delivering care congruent with Diabetes UK's recommendations, eg. employing strong clinical leadership and better systems and technology, described more positive experiences and felt they were able to provide a high standard of care despite the disruptions.

They were able to elucidate the following common themes.

- Use of technology

- Service structure

- Relationships with others

- Recognition and importance

- Momentum and opportunity

Some case studies are also presented in the paper.

The authors conclude and make recommendations for future practice. They emphasise that preparedness in the short term is important and identify areas of practice, including the following important points. Maintaining or reinstating multidisciplinary diabetes inpatient teams. Evidencing the impact of enhanced services employed during the first peak of the pandemic to learn for the future. Maintaining technological advances or putting new systems in place where they have previously been unable to. Putting in place better processes to tackle key challenges such as discharge and patient education. Prioritising the well-being of healthcare professionals to ensure there is time for rest and recovery. They also hint at the use of technology-based care forming part of a 'new normal' for diabetes care patients and that this may form part of the service going forward.

https://onlinelibrary.wiley.com/doi/full/10.1111/dme.14442?casa token=8Vwgw7FyeGUAAAAA\%3 AEULSAMaCPzGpAPOAGhJrnx2d5ujTbA3hO7NANk5SnXol-38AzQI 173JD-qR-PbFridAPy OemiraQ 


\section{Efficacy of telemedicine for persons with type 1 diabetes during Covid19 lockdown}

This open access article published in the Journal of Nutrition and Diabetes in January 2021 sought to determine the effectiveness of telemedicine in diabetes care after the national lockdown imposed in March 2020 by the Italian government. The research team retrospectively analysed data from continuous or flash glucose monitoring systems shared through different cloud/online/internetbased systems during the lockdown by subjects with type 1 diabetes and compared the data obtained 4 weeks before and 4 weeks after structured telephonic visit. Variables considered were mean glucose, time spent in target $(70-180 \mathrm{mg} / \mathrm{dl})$, hypoglycaemia $(<70 \mathrm{mg} / \mathrm{dl})$ and hyperglycaemia $(>180 \mathrm{mg} / \mathrm{dl})$, coefficient of variation, and length of sensor use. 71 participants were enrolled in the study

They found that in the 4 weeks following the telephonic visit there was an improvement of glycaemic control, which showed a significant reduction of mean glucose, an increase of the time spent in target and a reduction of time spent in hyperglycaemia No changes were observed regarding glucose variability, time spent in hypoglycaemia, and length of sensor use. Similar results were observed in subjects treated with multiple daily injections or continuous subcutaneous insulin infusion. They conclude that the use of telephonic review, pared with remote parameter measurement was an effective way to replace routine clinic visits and recommend this could be integrated into future care opening up new means of review for patients with diabetes.

\section{https://www.nature.com/articles/s41387-020-00147-8}

\section{Conclusion}

The NHS is still functioning in management, review and treatment of long-term chronic conditions despite the COVID-19 pandemic, but what is clear is that methods of consultation and monitoring have had to change due to the current situation. This is seen to be the case globally and not just a UK phenomenon. Suggestions are that we may not need to resort to the traditional, full face to face management in the future as overall, remote management has shown no decrease in satisfaction with care or in the achievement of positive outcomes despite the lack of patient attendance or changes to inpatient service delivery brought about by the COVID-19 pandemic. 